Generation and characterization of spirally polarized fields

This article has been downloaded from IOPscience. Please scroll down to see the full text article.

2009 J. Opt. A: Pure Appl. Opt. 11085708

(http://iopscience.iop.org/1464-4258/11/8/085708)

View the table of contents for this issue, or go to the journal homepage for more

Download details:

IP Address: 147.96.14.16

The article was downloaded on 11/06/2013 at 16:44

Please note that terms and conditions apply. 


\title{
Generation and characterization of spirally polarized fields
}

\author{
V Ramírez-Sánchez ${ }^{1}$, G Piquero ${ }^{1}$ and M Santarsiero ${ }^{2}$ \\ ${ }^{1}$ Departamento de Óptica, Facultad de Ciencias Físicas, Universidad Complutense de Madrid, \\ 28040 Madrid, Spain \\ 2 Dipartimento di Fisica, Università Roma Tre, and CNISM, Via della Vasca Navale 84, \\ 00146 Rome, Italy
}

Received 26 February 2009, accepted for publication 15 May 2009

Published 26 June 2009

Online at stacks.iop.org/JOptA/11/085708

\begin{abstract}
Recently introduced global parameters for describing the polarization of a beam are used to characterize spirally polarized fields, which include as particular cases azimuthally and radially polarized fields. Theoretical predictions about such global parameters are experimentally confirmed by generating beams with spirally polarized transverse patterns, by means of two different procedures.
\end{abstract}

Keywords: polarization, beam characterization

\section{Introduction}

Synthesis of optical beams with non-uniform distributions of polarization across the beam profile is a topic of growing interest in optics, both from theoretical and from applicative viewpoints. Several methods have been proposed in the literature to synthesize non-uniformly polarized beams [1-14]. Most of them are aimed at generating radially or azimuthally polarized beams. Just to quote some of them, we recall techniques using interferometric arrangements [5], sectored spatially varying retarders [6-8], space-variant wave plates based on stress birefringence [9], and polarization converters based on liquid-crystal devices [10-14].

As a particular case of non-uniformly totally polarized fields, spirally polarized beams (SPBs) [15-22] have attracted much interest in recent years. They present axially symmetric polarization patterns, with linear polarization at any point of the transverse profile and with the electric field lines being logarithmic spirals. SPBs include, as limiting cases, radially and azimuthally polarized beams [23, 24], and can always be thought of as the result of the linear superposition of two beams of such a kind [21]. Due to their high focusing capability [16-18], SPBs are used in several applications, such as optical tweezers, particle trapping, laser cutting, material processing, microscopy, etc [25-29].

On the other hand, for the characterization of nonuniformly polarized beams some overall parameters have been introduced in the literature. Examples are the average degree of polarization [30], the generalized degree of polarization [31], and others, which have been recently proposed for measuring the circular [32] and the radial and azimuthal polarization contents across the beam profile [33]. In the present work, the latter parameters are applied to the case of synthesized beams with spiral polarization distribution across a transverse plane. With this aim, two experimental techniques, making use of an azimuthal linear polarizer and a polarization converter, respectively, together with a polarization rotator, are presented.

\section{Preliminaries}

Let us begin by considering a non-uniformly polarized field represented by the following Jones vector [34] across a plane $z=$ constant: [15]

$$
\mathbf{E}(\mathbf{r})=f(r, \theta)\left(\begin{array}{c}
-\sin (\theta+\alpha) \\
\cos (\theta+\alpha)
\end{array}\right),
$$

where $\mathbf{r}=(r, \theta)$ is the position vector across the plane and $\alpha$ is a constant angle. Since the polarization state is not defined at the center of the profile, the function $f$ must be chosen in such a way that the amplitude vanishes at $r=0$. The structure in equation (1) describes a field whose polarization is linear at any point and symmetric around the propagation axis, as shown in figure 1 , even though its amplitude may depend on $\theta$. The electric field lines are logarithmic spirals whose growth parameter depends on the value of $\alpha$ [15], so that, by varying $\alpha$, different patterns of the polarization across the beam section are obtained, ranging from azimuthal (when $\alpha=0$ ) to radial (when $\alpha=\pi / 2$ ) polarization. If the function $f$ is independent 


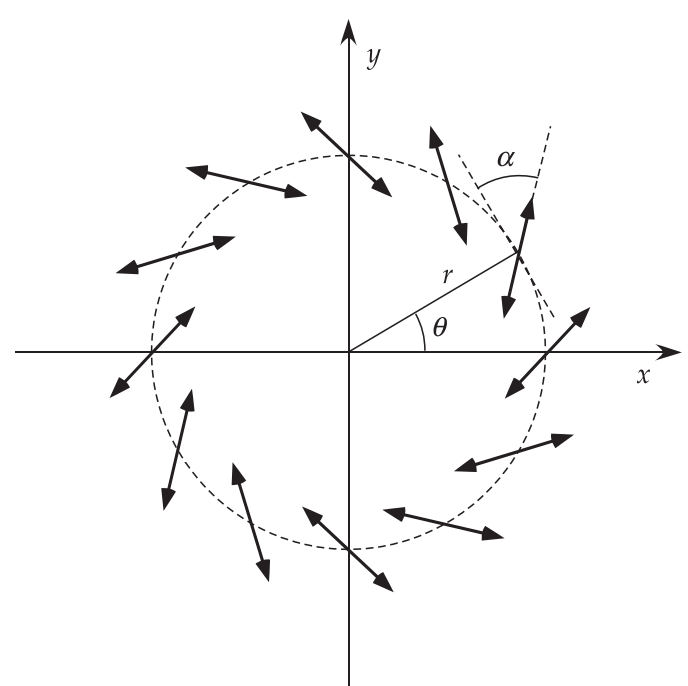

Figure 1. Polarization pattern of a spirally polarized field.

of $\theta$, the beam presents the same polarization pattern across any transverse plane in the paraxial approach [20].

Fields written as in equation (1) can always be expressed as the sum of a radially polarized term and an azimuthally polarized one, according to the form

$$
\mathbf{E}(\mathbf{r})=f(r, \theta)\left[-\sin \alpha\left(\begin{array}{c}
\cos \theta \\
\sin \theta
\end{array}\right)+\cos \alpha\left(\begin{array}{c}
-\sin \theta \\
\cos \theta
\end{array}\right)\right] .
$$

As is evident, the contributions of the radial and azimuthal parts will change on varying $\alpha$. This suggests that significant parameters for the characterization of this kind of profile will be those global parameters that give the radial and azimuthal polarization contents, respectively, across the transverse section of the beam [33].

Let us then recall the following parameters [33]:

$$
\begin{gathered}
\tilde{\rho}_{\mathrm{r}}=\frac{1}{P} \int_{0}^{\infty} \int_{0}^{2 \pi} \rho_{\mathrm{r}}(r, \theta) I(r, \theta) r \mathrm{~d} r \mathrm{~d} \theta \\
\tilde{\rho}_{\mathrm{a}}=\frac{1}{P} \int_{0}^{\infty} \int_{0}^{2 \pi} \rho_{\mathrm{a}}(r, \theta) I(r, \theta) r \mathrm{~d} r \mathrm{~d} \theta \\
\sigma_{\mathrm{r}}^{2}=\frac{1}{P} \int_{0}^{\infty} \int_{0}^{2 \pi}\left[\rho_{\mathrm{r}}(r, \theta)-\tilde{\rho}_{\mathrm{r}}\right]^{2} I(r, \theta) r \mathrm{~d} r \mathrm{~d} \theta \\
\sigma_{\mathrm{a}}^{2}=\frac{1}{P} \int_{0}^{\infty} \int_{0}^{2 \pi}\left[\rho_{\mathrm{a}}(r, \theta)-\tilde{\rho}_{\mathrm{a}}\right]^{2} I(r, \theta) r \mathrm{~d} r \mathrm{~d} \theta
\end{gathered}
$$

with

$$
P=\int_{0}^{\infty} \int_{0}^{2 \pi} I(r, \theta) r \mathrm{~d} r \mathrm{~d} \theta
$$

where $\rho_{\mathrm{r}}$ and $\rho_{\mathrm{a}}$ give the irradiance percentage of the radial or azimuthal component, respectively, of the field at each point across the beam profile. Therefore, $\tilde{\rho}_{\mathrm{r}}$ and $\tilde{\rho}_{\mathrm{a}}$ represent the radial and azimuthal polarization contents of a nonuniformly polarized beam profile, weighted with the values of its irradiance. On the other hand, $\sigma_{\mathrm{r}}^{2}$ and $\sigma_{\mathrm{a}}^{2}$ are the variances of $\tilde{\rho}_{\mathrm{r}}$ and $\tilde{\rho}_{\mathrm{a}}$, so they give information about the uniformity of the above polarization contents across the beam profile.
It is useful to write these parameters in terms of the local Stokes parameters $\left(s_{i}, i=0,1,2,3\right)$ as [33]

$$
\begin{aligned}
\tilde{\rho}_{\mathrm{r}}= & \frac{1}{2}+\frac{1}{2 P} \int_{0}^{\infty} \int_{0}^{2 \pi}\left[\cos (2 \theta) s_{1}(r, \theta)\right. \\
& \left.+\sin (2 \theta) s_{2}(r, \theta)\right] r \mathrm{~d} r \mathrm{~d} \theta, \\
\tilde{\rho}_{\mathrm{a}}= & \frac{1}{2}-\frac{1}{2 P} \int_{0}^{\infty} \int_{0}^{2 \pi}\left[\cos (2 \theta) s_{1}(r, \theta)\right. \\
& \left.+\sin (2 \theta) s_{2}(r, \theta)\right] r \mathrm{~d} r \mathrm{~d} \theta .
\end{aligned}
$$

In turn, the local Stokes parameters can be evaluated from the intensity patterns across the beam profile after the insertion of a suitably oriented quarter-wave plate and a linear polarizer having transmission axis at different angles with respect to the $x$-axis. With evident meaning of the involved symbols, we have [35]

$$
\begin{gathered}
s_{0}(r, \theta)=I_{0^{\circ}}(r, \theta)+I_{90^{\circ}}(r, \theta), \\
s_{1}(r, \theta)=I_{0^{\circ}}(r, \theta)-I_{90^{\circ}}(r, \theta), \\
s_{2}(r, \theta)=I_{45^{\circ}}(r, \theta)-I_{135^{\circ}}(r, \theta), \\
s_{3}(r, \theta)=I_{\lambda / 4,45^{\circ}}(r, \theta)-I_{\lambda / 4,135^{\circ}}(r, \theta) .
\end{gathered}
$$

As can be easily seen from the above expressions, for fields written as in equation (1) the parameters defined in equations (3)-(6) are independent of the field amplitude $f(r, \theta)$ and turn out to be

$$
\begin{aligned}
& \tilde{\rho}_{\mathrm{r}}=\frac{1}{2}-\frac{1}{2} \cos (2 \alpha), \\
& \tilde{\rho}_{\mathrm{a}}=\frac{1}{2}+\frac{1}{2} \cos (2 \alpha),
\end{aligned}
$$

and

$$
\sigma_{\mathrm{r}}^{2}=\sigma_{\mathrm{a}}^{2}=0 .
$$

Another useful overall parameter for characterizing non-uniformly polarized beams is $\tilde{\rho}_{\mathrm{c}}$, which represents the linear or circular polarization content across the beam profile, weighted with the values of the irradiance. It is defined in terms of the $s_{3}$ Stokes parameter as [32]

$$
\tilde{\rho}_{\mathrm{c}}=\frac{1}{P} \int_{0}^{\infty} \int_{0}^{2 \pi} \rho_{\mathrm{c}}(r, \theta) s_{0}(r, \theta) r \mathrm{~d} r \mathrm{~d} \theta
$$

with

$$
\rho_{\mathrm{c}}=\frac{s_{3}(r, \theta)}{s_{0}(r, \theta)} .
$$

The parameter $\tilde{\rho}_{\mathrm{c}}$ ranges from -1 for pure left-handed circularly polarized light to +1 for pure right-handed circularly polarized light, and the value $\tilde{\rho}_{\mathrm{c}}=0$ corresponds to a field with pure linear, possibly non-uniform, polarization, as is the case of spirally polarized fields.

\section{Experiment}

We synthesized two different beam profiles having the form given in equation (1). In both cases we used the optical setup shown in figure 2.

The light beam emerging from a He-Ne laser (SpectraPhysics 117A) is expanded, spatially filtered, and collimated by means of the collimation system (CS). The laser beam 


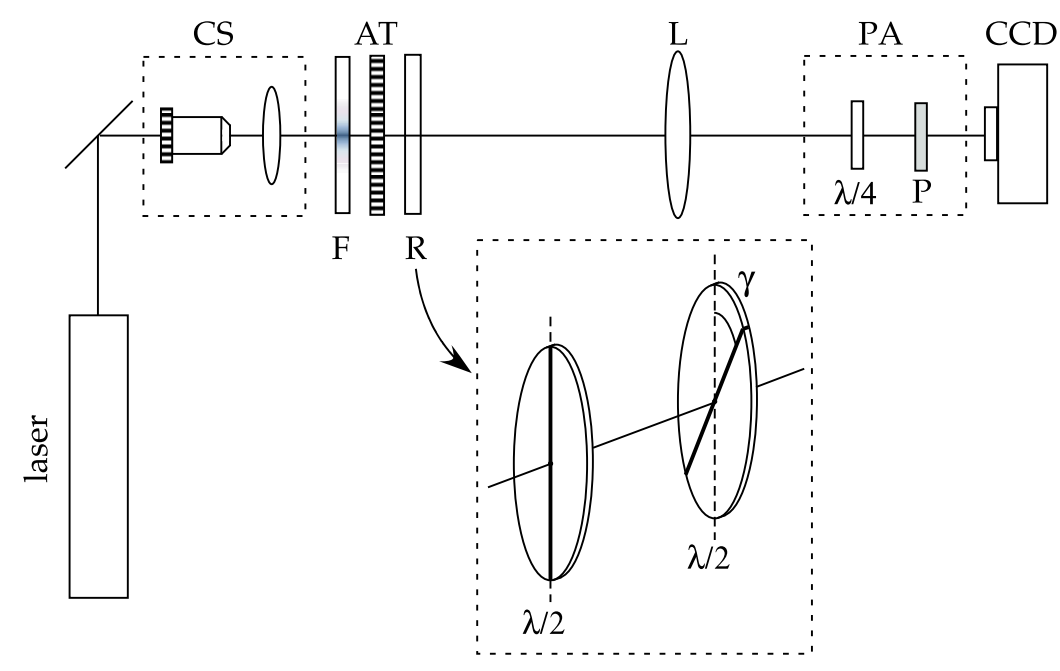

Figure 2. Experimental setup for the synthesis of spirally polarized fields. CS, collimation system; F, amplitude filter; AT, azimuthal polarization tool; $\mathrm{R}$, rotator; $\lambda / 2$, half-wave plate; $\mathrm{L}$, imaging lens; PA, polarization analyzer; $\lambda / 4$, quarter-wave plate; $\mathrm{P}$, linear polarizer.

is linearly polarized along the vertical $(y)$ direction. An amplitude filter $(\mathrm{F})$ is placed after $\mathrm{CS}$ to produce zero irradiance at the center of the beam. The analytical form of this filter is approximately $t(r)=\left[1-\exp (-r / w)^{4}\right]$, with $w \approx$ $1 \mathrm{~mm}$. The element AT represents a tool aimed at producing an azimuthally polarized field. We used two different tools in the two experiments, as we shall see in the following. After AT, a polarization rotator $(\mathrm{R})$ is placed, which locally rotates the electric field of the incident beam, then producing a spirally polarized field [21]. It consists of two half-wave plates, whose fast axes form the angle $\gamma$ with one another. It can be easily verified that the Jones matrix of this pair of plates is $[21,22]$

$$
\hat{R}=\left(\begin{array}{cc}
\cos (2 \gamma) & -\sin (2 \gamma) \\
\sin (2 \gamma) & \cos (2 \gamma)
\end{array}\right)
$$

and therefore it represents a rotation by $2 \gamma$ of the incident polarization.

The field emerging from the rotator is eventually imaged onto the CCD sensor of a camera by means of the lens (L). Just before the camera the polarization analyzer (PA), consisting in a quarter-wave plate $(\lambda / 4)$ and a linear polarizer $(\mathrm{P})$, is used for the measurement of the local Stokes parameters of the field across its transverse section.

In the first experiment, the azimuthal polarization tool consisted in a quarter-wave phase plate followed by a dichroic azimuthal linear polarizer (usually called an 'axis finder', from Edmund Optics). The wave plate, suitably oriented, converts the incident linear polarization into a right-handed circular one. Since the Jones matrix of the axis finder can be written as

$$
\hat{A}=\left(\begin{array}{cc}
\sin ^{2} \theta & -\cos \theta \sin \theta \\
-\cos \theta \sin \theta & \cos ^{2} \theta
\end{array}\right),
$$

disregarding the circularly symmetric amplitude factor produced by the transparency $F$, as well as any other constant factors, an output field of the type

$$
\mathbf{E}(\mathbf{r}) \propto \exp (\mathrm{i} \theta)\left(\begin{array}{c}
-\sin \theta \\
\cos \theta
\end{array}\right)
$$

is obtained. Finally, after the rotator, the Jones vector of the synthesized field turns out to be

$$
\mathbf{E}(\mathbf{r}) \propto \exp (\mathrm{i} \theta)\left(\begin{array}{c}
-\sin (\theta+2 \gamma) \\
\cos (\theta+2 \gamma)
\end{array}\right)
$$

which is exactly of the form in equation (1), provided that $\gamma=$ $\alpha / 2$ is chosen. Note that, due to the presence of the quarterwave plate, the irradiance profile of the field is rotationally symmetric.

Experimental results are obtained by changing $\gamma$ from $0^{\circ}$ to $90^{\circ}$, i.e. from azimuthal to azimuthal polarization, passing through radial polarization, occurring when $\gamma=45^{\circ}$. In this way, several non-uniformly linearly polarized fields are generated with different radial and azimuthal polarization contents. As can be easily understood, the irradiance profile of the synthesized field does not depend on the value of the parameter $\gamma$. The experimental irradiance profile for $\alpha=20^{\circ}$ is reported in figure 3 , where the polarization states at several points of the profile are also shown. Similar patterns are obtained for different values of $\alpha$.

In figure 4 the experimental values of the parameters $\tilde{\rho}_{\mathrm{r}}$ and $\tilde{\rho}_{\mathrm{a}}$ are represented versus the rotation angle $\alpha$, together with the theoretical curves calculated from equations (14) and (15). The average values of the dispersions, $\sigma_{\mathrm{r}}^{2}$ and $\sigma_{\mathrm{a}}^{2}$, are both equal to $0.0114 \pm 0.0001$.

To get further information about the spirally polarized profile, the circular polarization content $\tilde{\rho}_{\mathrm{c}}$ has been evaluated by means of equation (17). The experimental values are presented in figure 5 (dots) as functions of the rotation angle $\alpha$. The deviation from the theoretical prediction (zero) shows a nonideal behavior of the axis finder. Nevertheless, the circular polarization content is rather small, resulting in an average value of $0.13 \pm 0.01$.

In the second experiment, the azimuthal polarization tool consisted of an Arcoptix polarization converter, which locally rotates an incident linear polarization using a liquid-crystal device [10]. In such a case the Jones matrix of the polarization 


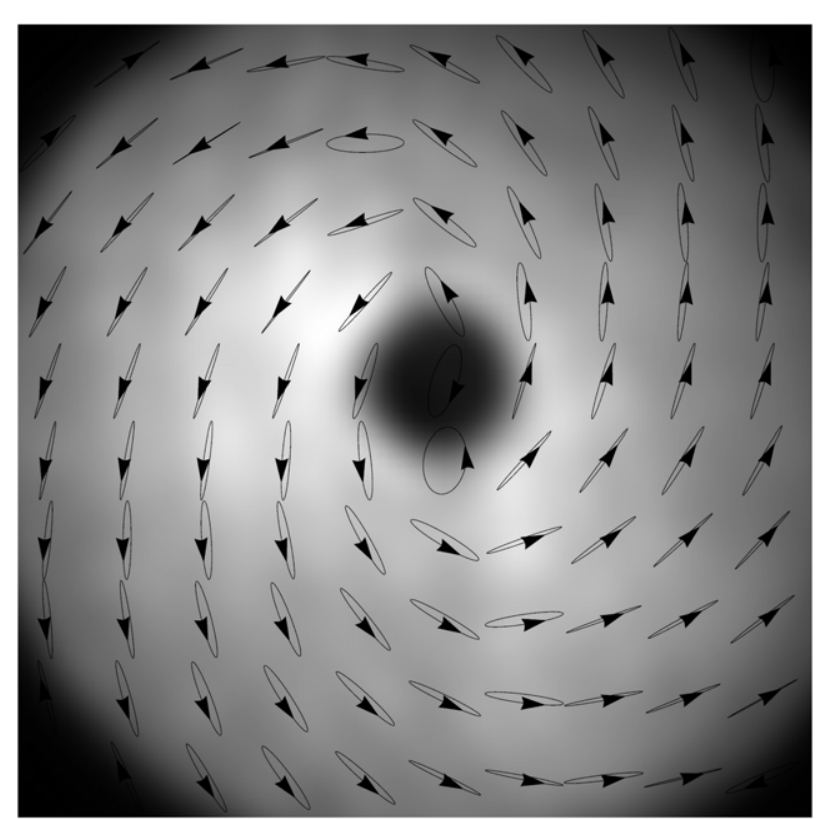

Figure 3. Experimental irradiance and polarization pattern in the first experiment for $\alpha=20^{\circ}$. The transverse size of the shown profile is about $1 \mathrm{~cm}$.

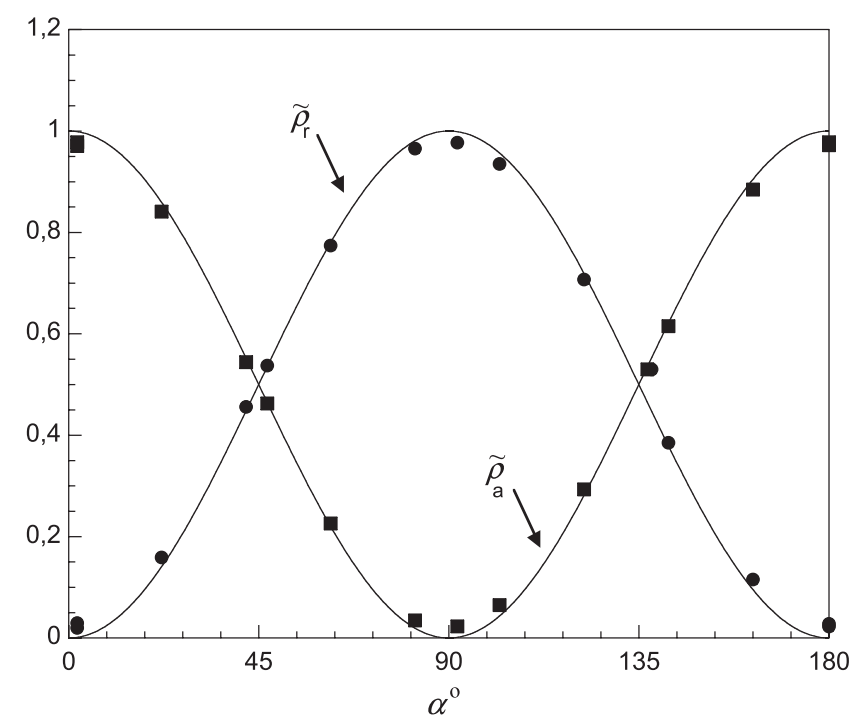

Figure 4. Experimental values of parameters $\tilde{\rho}_{\mathrm{r}}$ (circles) and $\tilde{\rho}_{\mathrm{a}}$ (squares) versus the field rotation angle, $\alpha$, together with their theoretical values (solid curves), for the first experiment (fields synthesized by means of an axis finder).

converter can be evaluated as

$$
\hat{A}=\left(\begin{array}{cc}
\cos \theta & -\sin \theta \\
\sin \theta & \cos \theta
\end{array}\right),
$$

so that the output field is given by

$$
\mathbf{E}(\mathbf{r}) \propto\left(\begin{array}{c}
-\sin \theta \\
\cos \theta
\end{array}\right)
$$

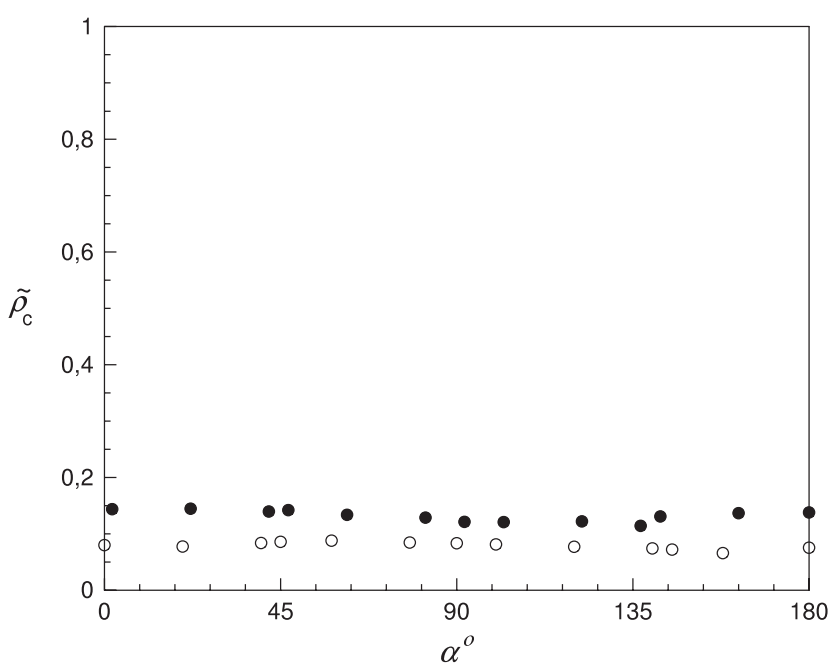

Figure 5. Experimental values of parameter $\tilde{\rho}_{\mathrm{c}}$ versus $\alpha$. Dots correspond to the first experiment and circles to the second one.

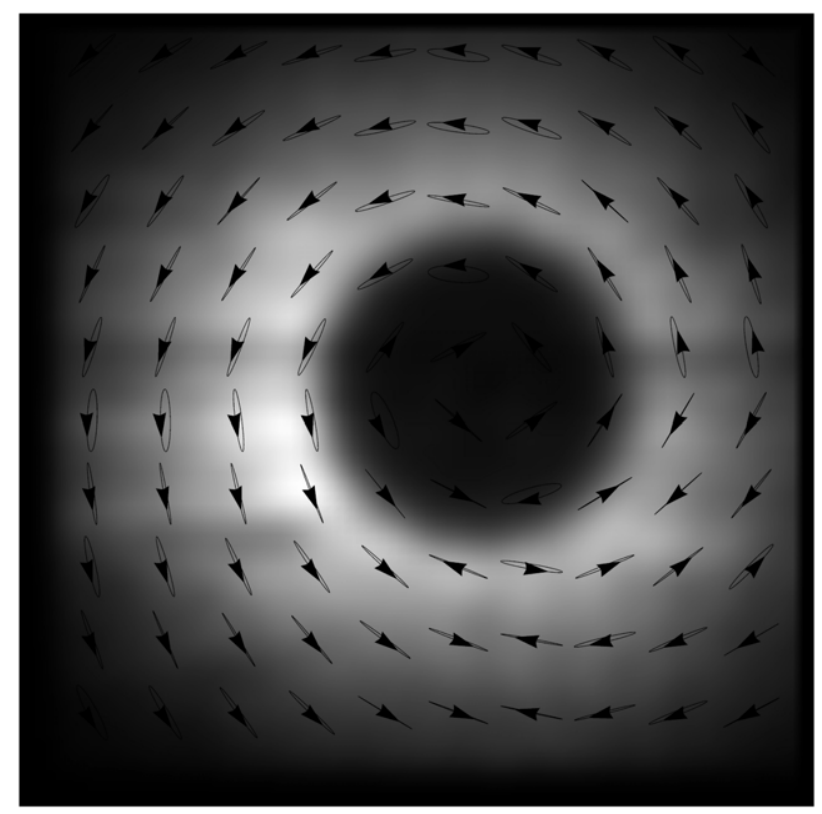

Figure 6. Experimental irradiance and polarization pattern in the second experiment for $\alpha=20^{\circ}$. The transverse size of the shown profile is about $0.5 \mathrm{~cm}$.

Finally, after the rotator, the electric field turns out to be

$$
\mathbf{E}(\mathbf{r}) \propto\left(\begin{array}{c}
-\sin (\theta+2 \gamma) \\
\cos (\theta+2 \gamma)
\end{array}\right)
$$

which is similar to equation (22), but without the phase factor $\exp (\mathrm{i} \theta)$

The irradiance profile and the polarization pattern for $\alpha=$ $20^{\circ}$ is shown in figure 6 , while in figure 7 the experimental values of $\tilde{\rho}_{\mathrm{r}}$ and $\tilde{\rho}_{\mathrm{a}}$ are plotted versus $\alpha$ (solid curves represent theoretical values). The average values of the variances $\sigma_{\mathrm{r}}^{2}$ and $\sigma_{\mathrm{a}}^{2}$ are both equal to $0.049 \pm 0.002$. In this case the variances are slightly higher than for the first experiment, showing a less 


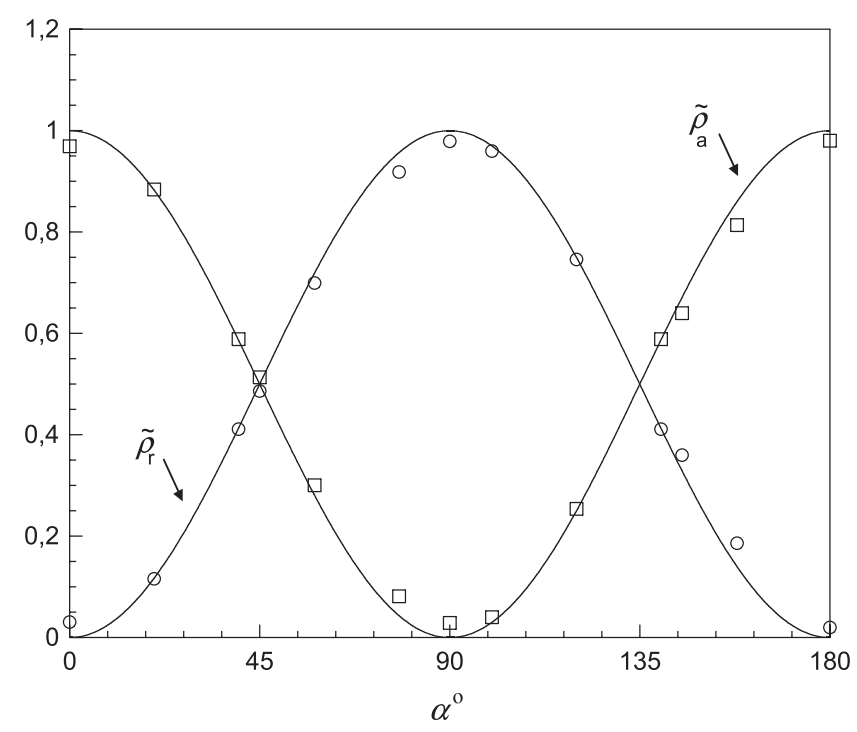

Figure 7. Experimental values of parameters $\tilde{\rho}_{\mathrm{r}}$ (circles) and $\tilde{\rho}_{\mathrm{a}}$ (squares) versus the field rotation angle, $\alpha$, together with their theoretical values (solid curves), for the second experiment (fields synthesized by means of liquid-crystal polarization converter).

uniform distribution of the radial and azimuthal polarization contents across the beam profile.

The experimental values of the circular polarization content $\tilde{\rho}_{\mathrm{c}}$ are shown in figure 5 (circles) as functions of the rotation angle $\alpha$. Even in this case a slight deviation from the theoretical value (zero) can be appreciated, resulting in an average value of $0.08 \pm 0.01$. As can be seen from figure 5, the values are lower for the second experiment than for the first one, so the synthesized beam produced using the polarization converter is globally more linearly polarized than the other one. Such results give an account of the differences between the polarization patterns shown in figures 3 and 6 .

\section{Conclusions}

Two experimental procedures have been used to synthesize beams with spirally polarized transverse patterns. The techniques make use of an axis finder and a polarization converter, respectively, together with a polarization rotator. The generated fields have been characterized by means of some recently introduced global parameters, aimed at characterizing non-uniformly totally polarized light beams. Experimental results show good agreement between theory and experiment for both the techniques.

\section{Acknowledgments}

This work has been supported by the Ministerio de Educación y Ciencia of Spain, project FIS2007-63396, and project CCG07UCM/ESP-3070.

M Santarsiero wishes to thank R Martínez-Herrero, P Mejías, J Serna, G Piquero and V Ramírez-Sánchez for their kind hospitality during his stay at Universidad Complutense de Madrid.

\section{References}

[1] Nesterov A V and Niziev V G 2000 Laser beams with axially symmetric polarization J. Phys. D: Appl. Phys. 33 1817-22

[2] Niv A et al 2003 Formation of linearly polarized light with axial symmetry by use of space-variant subwavelength gratings Opt. Lett. 28 510-2

[3] Volpe G and Petrov D 2004 Generation of cylindrical vector beams with few-mode fibers excited by Laguerre-Gaussian beams Opt. Commun. 237 89-95

[4] Niu Ch et al 2005 A new method for generating axially-symmetric and radially-polarized beams $J$. Phys. D: Appl. Phys. 38 827-32

[5] Tidwell S C, Ford D H and Kimura W D 1990 Generating radially polarized beams interferometrically Appl. Opt. 29 2234-9

[6] Quabis S, Dorn R and Leuchs G 2005 Generation of a radially polarized doughnut mode of high quality Appl. Phys. B 81 597-600

[7] Machavariani G, Lumer Y, Moshe I, Meir A and Jackel S 2008 Spatially-variable retardation plate for efficient generation of radially and azimuthally polarized beams $O p t$. Commun. $281732-8$

[8] Kawauchi H, Kozawa Y, Sato S, Sato T and Kawakami S 2008 Simultaneous generation of helical beams with linear and radial polarization by use of a segmented half-wave plate Opt. Lett. 33 399-401

[9] Spilman A K and Brown T G 2007 Stress birefringent, space-variant wave plates for vortex illumination Appl. Opt. 46 61-6

[10] Stalder M and Schadt M 1996 Linearly polarized light with axial symmetry generated by liquid-crystal polarization converters Opt. Lett. 21 1948-50

[11] Bentley J B, Davis J A, Bandress M A and Gutierrez-Vega J C 2006 Generation of helical Ince-Gaussian beams with a liquid-cystal display Opt. Lett. 31 649-51

[12] Ren $\mathrm{H}$ and Wu S T 2007 Liquid-cystal-based linear polarization rotator Appl. Phys. Lett. 90121123

[13] Tzeng Y Y, Ke S W, Ting C L, Fuh A Y G and Lin T H 2008 Axially symmetric polarization converters based on photo-aligned liquid crystal films Opt. Express 16 3768-75

[14] McEldowney S C, Shemo D M and Chipman R A 2008 Vortex retarders produced from photo-aligned liquid crystal polymers Opt. Express 16 7295-308

[15] Gori F 2001 Polarization basis for vortex beams J. Opt. Soc. A $181612-7$

[16] Youngworth K S and Brown T G 2000 Focusing of high numerical aperture cylindrical-vector beams Opt. Express $777-87$

[17] Borghi R, Santarsiero M and Alonso M A 2005 Highly focused spirally polarized beams J. Opt. Soc. A 22 1420-31

[18] Hao B and Leger J 2008 Numerical aperture invariant focus shaping using spirally polarized beams Opt. Commun. 281 1924-8

[19] Ramírez-Sánchez V and Piquero G 2008 The beam quality parameter of spirally polarized beams J. Opt. A: Pure Appl. Opt. 10125004

[20] Gori F 2008 Partially correlated sources with complete polarization Opt. Lett. 33 2818-20

[21] Zhan Q and Leger J R 2002 Focus shaping using cylindrical vector beams Opt. Express 10 324-31

[22] Rao L, Pu J, Chen Z and Yei P 2009 Focus shaping of cylindrically polarized vortex beams by a high numerical-aperture lens Opt. Laser Technol. 41 241-6

[23] Martínez-Herrero R, Mejías P M and Bosch S 2008 On the vectorial structure of non-paraxial radially polarized light fields Opt. Commun. $2813046-50$ 
[24] Martínez-Herrero R and Mejías P M 2008 Propagation of light fields with radial or azimuthal polarization distribution at a transverse plane Opt. Express 16 9021-33

[25] Dorn R, Quabis S and Leuchs G 2003 Sharper focus for radially polarized light beams Phys. Rev. Lett. 91233901

[26] Zhan Q 2004 Trapping metallic Rayleigh particles with radial polarization Opt. Express 12 3377-82

[27] Niziev V G and Nesterov A V 1999 Influence of beam polarization on laser cutting efficiency J. Phys. D: Appl. Phys. 32 1455-61

[28] Liu Y, Cline D and He P 1999 Vacuum laser acceleration using a radially polarized $\mathrm{CO}_{2}$ laser beam $\mathrm{Nucl}$. Instrum. Methods Phys. Rev. A 424 296-303

[29] Novotny L, Beversluis M R, Youngworth K S and Brown T G 2001 Longitudinal field modes probed by single molecules Phys. Rev. Lett. 86 5251-4

[30] Piquero G, Movilla J M, Mejías P M and Martínez-Herrero R 1999 Degree of polarization of non-uniformly partially polarized beams: a proposal $O p t$. Quantum Electron. 31 223-5

[31] Mejías P M, Martínez-Herrero R, Piquero G and Movilla J M 2002 Parametric characterization of the spatial structure of non-uniformly polarizad laser beams Prog. Quantum Electron. 26 65-130

[32] Martínez-Herrero R, Mejías P M and Piquero G 2006 Overall parameters for the characterization of non-uniformly totally polarized beams Opt. Commun. 265 6-10

[33] Martínez-Herrero R, Mejías P M, Piquero G and Ramírez-Sánchez V 2008 Global parameters for characterizing the radial and azimuthal polarization content of totally polarized beams Opt. Commun. 281 1976-80

[34] Brosseau C 1998 Fundamentals of Polarized Light (New York: Wiley)

[35] Born M and Wolf E 1999 Principles of Optics 7th (expanded) edn (Cambridge: Cambridge University Press) 